\title{
Vitiligo labial associé à une pathomimie
}

\author{
M. Fricain (Paris), P. Weidmann (Paris), Y. Roche (Paris), J.C. Fricain (Bordeaux)
}

Le vitiligo est une leucodermie affectant 0,5 à $1 \%$ de la population mondiale. Il n'y a pas de différence de prévalence concernant l'âge, le sexe ou le type de peau. La disparition des mélanocytes entraine une hypopigmentation localisée en plaques symétriques blanches ivoire, à bords nets souvent hyperpigmentés. Les lésions sont le plus souvent retrouvées au niveau des zones découvertes, de frottement et des extrémités. L'évolution des lésions est imprévisible, alternant des phases de développement et de quiescence. Le mécanisme physiopathologique est mal connu, il s'agirait d'une pathologie auto immune avec une prédisposition héréditaire sur un terrain psychologique affaibli. On le retrouve associé dans d'autres affections auto immunes : l'insuffisance surrénalienne, les pathologies thyroïdiennes et la maladie de Biermer (Nagarajan et al (2015)). Le cas clinique rapporté est celui d'une jeune femme de 17 ans, qui présentait une dépigmentation de la lèvre supérieure apparue en octobre 2016. Initialement, la lésion affectait l'hémi lèvre supérieure gauche. Le dermatologue avait posé le diagnostic de vitiligo et instauré un traitement par vitamine $C$ et acide folique, suivi pendant un mois, sans résultat. En juillet 2017 la patiente a consulté en pathologie de la muqueuse buccale car la lésion s'était étendue à l'ensemble de la lèvre supérieure avec atteintes de la commissure labiale gauche et cutanée en regard. L'interrogatoire a révélé un mordillement chronique des lèvres. L'examen de la muqueuse buccale a mis en évidence une dépigmentation linéaire de du bord vermillon de la lèvre supérieure avec renforcement pigmentaire périphérique. L'examen cutané a révélé une plage dépigmentée centimétrique de l'auriculaire de la main droite. Un bilan biologique incluant thyréostimuline, anticorps anti thyroglobuline et anticorps antithyroperoxydase a été prescrit de façon systématique. Il n'a pas révélé d'anomalie. Le traitement prescrit était : tacrolimus à $0,1 \%$ en application locale biquotidienne et arrêt de la pathomimie. Le vitiligo des muqueuses buccales est rare. Il a essentiellement été décrit en Inde où la maladie est endémique (Nagarajan et al (2015)). L'atteinte des muqueuses orales concernerait 55\% des patients et la lèvre serait touchée dans près d'un cas sur 2 dans cette population. Le cas présenté concernait une patiente originaire d'Afrique du nord. Les études concernant le traitement du vitiligo labial ont été menées uniquement sur le traitement chirurgical : micropigmentation et greffes de mélanocytes semblent avoir le plus fort taux de succès (Gupta et al (2006)). Rodrigues et al (2017) ont conseillé une application locale biquotidienne de tacrolimus 0,1\% pour les affections de la face et des zones intertrigineuses. De par son mode d'action, le tacrolimus a une activité immunosuppressive et pourrait favoriser la pigmentation de la muqueuse orale (Fricain et al 2005). Dans le cas présenté, un traitement par tacrolimus a été instauré dans un premier temps. Un traitement chirurgical par greffe de mélanocytes ou un traitement cosmétique sera proposé en cas d'échec du traitement local. Bien que rare, le vitiligo de la muqueuse buccale ne doit pas être ignoré du chirurgien oral qui devra collaborer avec le dermatologue pour définir le traitement adapté. 